\title{
Real-Time Intelligent Decision Support System for Bridges Structures Behavior Prediction
}

\author{
Hélder Quintela ${ }^{1}$, Manuel Filipe Santos ${ }^{1}$, Paulo Cortez ${ }^{1}$ \\ ${ }^{1}$ Department, University of Minho \\ Campus de Azurém \\ 4800-058 Guimarães, Portugal \\ \{hquintela,mfs,pcortez\}@dsi.uminho.pt
}

\begin{abstract}
There is an increasing need of deploying automatic real-time decision support systems for civil engineering structures, making use of prediction models based in Artificial Intelligence techniques (e.g., Artificial Neural Networks) to support the monitoring and prediction activities. Past experiments with Data Mining (DM) techniques and tools opened room for the development of such a real-time Decision Support System. However, it is necessary to test this approach in a real environment, using real-time sensors monitoring. This study presents the development of prediction models for structures behavior and a novel architecture for operating in a real-time system.
\end{abstract}

Keywords: Knowledge Discovery in Databases, Data Mining, Decision Trees, Neural Networks, Intelligent Decision Support Systems, Civil Engineering Structures

\section{Introduction}

In the field of Civil Engineering the increasement on the number of bridges in service, and the appearance of widespread failures has highlighted the importance of condition assessment using a new generation of tools that incorporate technology into real-time monitoring and decision support using expert systems - a new generation of Bridge Management Systems (BMS) that can help in maintenance, safety and management.

A bridge maintenance policy needs to incorporate multidisciplinary subjects from structural engineering, economics and computer science [1]. The future in inspection and condition assessment shows the need of incorporating in BMS models based in Artificial Intelligence, such as Artificial Neural Networks (ANN), for prediction of condition deterioration, developed using data collected: (i) in real time by sensors in the structure, (ii) in visual inspections by experts, (iii) in destructive and nondestructive tests. However the development of such models may not be an easy task using statistical techniques for historical data analysis, due to the high complexity of data and relations between several variables [2].

Regarding that, the application of Data Mining techniques (DM) to analyze civil engineering data has gained an increasing interest, due to the intrinsic characteristics 
such as ability to deal with non-linear relationships. The results attained by previous studies (some of them presented in this paper in the Previous Work section) are encouraging and open room for the development of Intelligence Decision Support Systems for Bridge Management Systems.

According to Supratim and Vankayala [3], Real-Time Business Intelligence systems are gaining an increasing attention from the research community. Indeed, developing and maintaining these systems is a challenging task, with a critical issue that lies in assuring that these systems can really work with an almost zero latency.

Tools for support decision making processes and problem-solving activities have proliferated and evolved over the last decades from applications based in spreadsheet software, to decision-support systems incorporating optimization models, and finally to systems with components from artificial intelligence and statistics [4].

This paper presents the second stage of a bigger project, called SIISEC - an Intelligent System for Civil Engineering Structures, with the goal of deploying forecasting models using Knowledge Discovery from Databases (KDD)/Data Mining technologies for structure damage detection. In particular, the DM techniques considered include: Artificial Neural Networks (ANNs) and Decision Trees (DTs).

This paper is organized as follows: first, is presented an overview of some experiments with simple structural elements using DM techniques; then the experiments with a structure prototype are described, being the results analyzed in terms of several criteria; next, is presented a novel architecture for a new Intelligent Decision Support System for structures monitoring; finally, closing conclusions are drawn and future research activities are depicted.

\section{Previous Work}

The first experiments of the SIISEC project were centered at the development of predicting models for: (i) ultimate resistance of steel beams subjected to concentrated loads, and (ii) critical shear stress in tapered plate girders.

In the conception and design of civil engineering structures several factors should be considered: aesthectics, functionality, deformability, durability, resistance and cost. In general, this exercise is conditioned to the search of the safest solution with the lowest cost. This concern, associated to the evolution of the materials' properties and of the computational tools, led to the use of more refined design methods. However, the error presented by the current design formulas is significant due to: the influence of several independent parameters in the behavior; the insufficient number of experimental data that allows a parametric analysis; and the calibration of simplified models.

While parametric analysis is an intensive and hard work, the construction of models using DM techniques in a KDD process, could be used to induce predictive models in a more flexible and efficient fashion. Hence, the following studies, opened room for the development of the study presented in this paper, since the accuracy of the proposed DM models are better than the actual analytical formulations, enabling the development of models not for isolated elements of a civil structure (e.g., beams), but for the structure (e.g., bridge) as a whole. 


\subsection{Prediction of ultimate resistance of steel beams}

The models developed to predict the ultimate resistance of steel beams subjected to concentrated loads based in ANN presented a better accuracy that the design formula proposed by Roberts [5] as presented in Table 1. Model A is adjusted to beams with a web thickness lower or equal to 2.12 and Model B for beams with a web thickness higher.

In previous work [6] we have introduced a clustering approach [7], in order to search and explore some kind of homogeneity existing in the data set, producing in this way predicted models with a better degree of accuracy - what is a main issue -, to assure a high level of safety and usability. The proposed approach includes a macroanalysis in the data mining step of the KDD process, using a Kohonen ANN [8], to segment the available examples.

Table 1. Models' performance for ultimate resistance prediction.

\begin{tabular}{ccccc}
\hline & \multicolumn{2}{c}{ Model A } & \multicolumn{2}{c}{ Model B } \\
\hline & $A N N$ & Roberts & ANN & Roberts \\
Mean Error (\%) & 9,9 & 19,3 & 6,6 & 15,3 \\
& & & & \\
Standard & \multicolumn{2}{c}{3,32} \\
Deviation & \multicolumn{2}{c}{5,11} & \multicolumn{2}{c}{3,32} \\
$(\%$ mean error) & & & \multicolumn{2}{c}{} \\
\hline
\end{tabular}

\subsection{Prediction of ultimate shear resistance of non-prismatic tapered plate} girders

Tapered plate girders with slender webs are used in steel and composite structures, when there is necessity of large span lengths, and/or when the structure is required to bear heavy loads. The design of this kind of structures requires in-depth study of their buckling to ensure their correct behavior, both in situations close to collapse and in service situations.

The models proposed were developed using the clustering approach presented before and based in ANNs. 
Table 2. Models' performance for ultimate shear prediction

Model A

\begin{tabular}{ccc}
\hline Error & ANN & Zárate \\
\hline MAD & 0,658846 & 0,87 \\
SSE & 38,0461 & 22,7292 \\
MSE & 1,415938 & 0,8742 \\
RMSE & 1,189932 & 0,934987 \\
\hline
\end{tabular}

Model B

\begin{tabular}{ccc}
\hline Error & ANN & Zárate \\
\hline MAD & 0,223529 & 0,566471 \\
SSE & 1,9988 & 13,8585 \\
MSE & 0,11757647 & 0,815206 \\
RMSE & 0,34289426 & 0,902888 \\
\hline
\end{tabular}

In [9], it was used a dataset with 176 girders with geometric parameters and the results of buckling coefficient and of the critical shear stress, using the Zárate Model and the Finite Element Method. One of the lessons learned stressed that even with a small number of samples, the ANN are an applicable machine learning paradigm in prediction tasks, since the available data is representative of the universe in study.

The models (Table 2) were compared with the study of Zárate [10], using some of the widespread measures used in regression tasks. The accuracy of the predicting models generated making use of machine learning techniques in a KDD process proven to be better for Model B that the previous model proposed by Zárate, while for Model A the results are almost the same.

\section{Data Mining Models for Structures Behavior Prediction}

The main questions addressed in this present work are:

- What was the weight that the structure was subjected?;

- What was the point on the structure where the load was placed?;

- What are the values for dislocation and deformation of the structure, taking in account the weight of the load and the point where the load was placed in the structure?

\subsection{Materials and Methods}

The dataset used in this study considers 1050 experiments in a prototype of a civil structure with 7 sensors for real-time collection of data, with 9 variables (Table 3). The sensors were used for deformation and dislocation measuring when the structure was subjected to a load.

According to the Figure 1, deformation sensors were placed in positions $\boldsymbol{T}_{\boldsymbol{1}}$ (point $2250 \mathrm{~mm}$ ), $\boldsymbol{T}_{2}$ (point $3250 \mathrm{~mm}$ ) and $\boldsymbol{T}_{4}$ (point $1250 \mathrm{~mm}$ ); and the extensometers for dislocation measuring were placed in positions $\boldsymbol{E} \boldsymbol{X} \boldsymbol{T}_{1}$ (point $2850 \mathrm{~mm}$ ), $\boldsymbol{E} \boldsymbol{X} \boldsymbol{T}_{2}$ (point $3250 \mathrm{~mm}$ ), $\boldsymbol{E X T}_{3}$ (point $3700 \mathrm{~mm}$ ) and $\boldsymbol{E X X}_{4}$ (point $1250 \mathrm{~mm}$ ). 


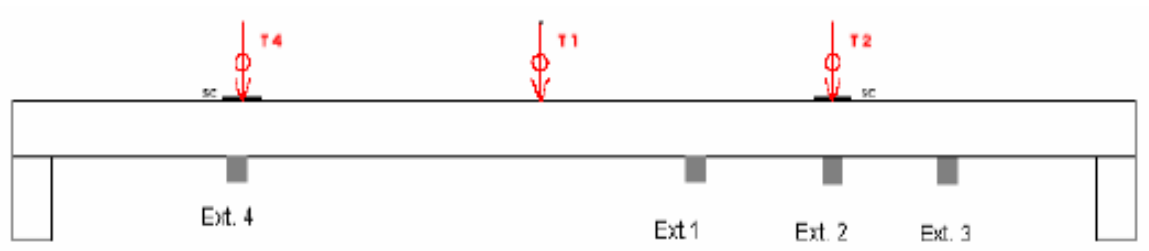

Fig. 1. Prototype of a civil structure with a length of 4.5 meters, with sensors location - $\boldsymbol{T}_{\boldsymbol{i}}$ are the deformation sensors and $\boldsymbol{E} \boldsymbol{X} \boldsymbol{T}_{\boldsymbol{j}}$ are the extensometers.

During the experiments were placed loads of 45 and $77 \mathrm{~kg}$ in the following points: $250 \mathrm{~mm}, 1250,2250 \mathrm{~mm}, 3250 \mathrm{~mm}, 4250 \mathrm{~mm}$, and when the structure was stabilized the data of deformation and dislocation values were collected to the database.

Table 3. Sensors variables and domain of values.

\begin{tabular}{cccc}
\hline Name & Min & Max & Mean \\
\hline $\mathrm{T}_{1}$ & 2.82 & 3.45 & 3.01 \\
$\mathrm{~T}_{2}$ & 2.05 & 3.06 & 2.69 \\
$\mathrm{~T}_{4}$ & 2.47 & 3.02 & 2.66 \\
$\mathrm{EXT}_{1}$ & 1120.4 & 1146.9 & 1127.4 \\
$\mathrm{EXT}_{2}$ & 1445.4 & 1476.2 & 1453.5 \\
$\mathrm{EXT}_{3}$ & 1291.1 & 1309.3 & 1295.9 \\
$\mathrm{EXT}_{4}$ & 799.8 & 960.5 & 928.8 \\
\hline
\end{tabular}

The analysis of the domain of values of the $\boldsymbol{T}$ and $\boldsymbol{E X} \boldsymbol{T}$ sensors indicates that the higher scale of the last ones could influence the results in the modeling phase. However, in the pre-processing tasks it was decided to keep the original values, and evaluate the results of the deployed models in the DM phase before any rescaling activity.

\subsection{What was the weight that the structure was subjected?}

The first DM experiments intend to develop a classification model that can predict the value of the weight that the structure was subjected; using the values of the sensors and the point where the load was applied. It was decided to use two techniques: DTs [11] and ANNs [12], as available in the SAS Enterprise Miner environment [13]. 
When using the DTs technique the split criteria used was the Gini Reduction. The obtained model (Figure 2) presents 7 leaves and a misclassification rate of 0 . The most important variable with an importance degree of 0.569 is the $E X T_{1}$ sensor, located almost in the middle of the structure, measuring the deformation of the structure when a load is applied.

In the ANN modelling, the popular Multi-Layer Perceptron (MLP) architecture [14] was adopted, where the number of hidden nodes in the hidden layer was automatically set by the SAS tool, and the accuracy was estimated using the Holdout method by randomly dividing the available data into three exclusive partitions: training, validation and testing.

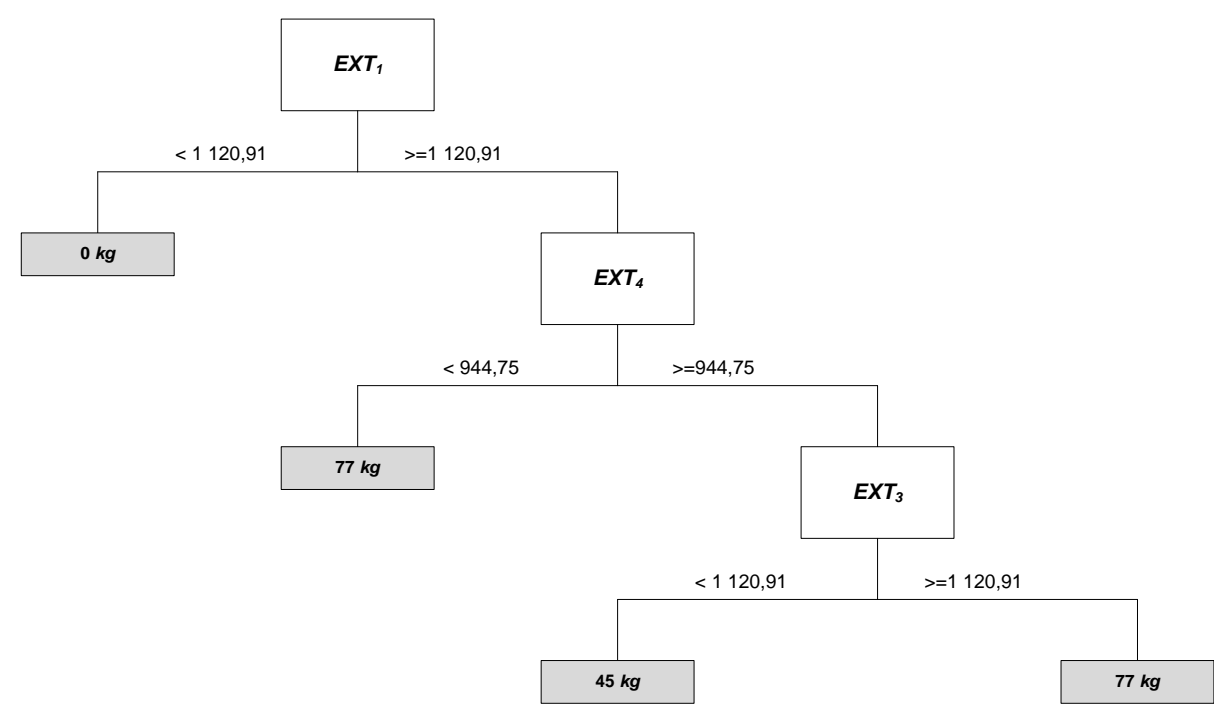

Fig. 2. Example of the Decision Tree based model for classification of the weight.

The final ANN based model for weight prediction presents architecture of 6 hidden nodes in the hidden layer, with a Maximum Absolute Error of 0.13. For evaluation of the two proposed models was used the Confusion Matrix [15] (Table 4) and calculated the Precision measure.

Table 4. Confusion Matrix for Decision Tree and Artificial Neural Network based models.

\begin{tabular}{cccc}
\hline Desired/Predicted & $\mathbf{0}$ & $\mathbf{4 5}$ & $\mathbf{7 7}$ \\
\hline $\mathbf{0}$ & 102 & 0 & 0 \\
$\mathbf{4 5}$ & 0 & 102 & 0 \\
$\mathbf{7 7}$ & 0 & 0 & 96 \\
\hline
\end{tabular}


Since the two models have an accuracy of $100 \%$, they can be used in this task, but the DT presents the advantage of easy understanding by civil engineering field specialists, which can evaluate and validate the captured knowledge, while the ANN based model is like a black box.

\subsection{What was the point on the structure where the load was placed?}

In order to plan the interventions on a structure could be important to know where the impact was applied. Similar to the previous task, both DTs and ANNs were used. However the results after several experiments, with different parameters and architectures, were not enough to validate this approach, since the error is very high.

\subsection{What are the values for dislocation and deformation of the structure?}

For the last question addressed in the DM phase, it was decided to use the ANN technique, to generate a model that predicts the value for dislocation $\left(T_{i}\right)$ and deformation $\left(E X T_{j}\right)$. It was adopted the following framework: (i) a model $\mathbf{A}$ with 2 inputs (weight and point) and 7 outputs ( $T_{i}$, and $\left.E X T_{j}\right)$; (ii) a model $\mathbf{B}$ with 2 inputs (weight and point) and 4 outputs $\left(E X T_{j}\right)$, rejecting the $T$ variables; and (iii) a model C with 2 inputs (weight and point) and 3outputs $\left(T_{i}\right)$, rejecting the $E X T$ variables. The best predictive performance was attained by the model A. Table 5 presents the predictive performance in test set for all the output variables of model A, using the Root Mean Squared Error (RMSE) measure (Equation 1). The RMSE is one of the most commonly used measures on regression tasks. This metric, which will be adopted in this work, has the property of being more sensitive to high error values.

$$
\sqrt{\frac{\left(a_{1}-c_{1}\right)^{2}+\left(a_{2}-c_{2}\right)^{2}+\ldots+\left(a_{n}-c_{n}\right)^{2}}{\left(a_{1}-\bar{c}\right)^{2}+\left(a_{2}-\bar{c}\right)^{2}+\ldots+\left(a_{n}-\bar{c}\right)^{2}}}
$$

The results show that the $E X T_{4}$ variable presents the higher error and that can be due to a noise problem of the sensor in data collection during the experiments, what can influence the value of the overall RMSE error. To test that hypothesis the variable was rejected, and the new model presents a better overall RMSE Error of 3.47, while the errors for the other variables are almost the same. 
Table 5. The predictive test performance.

\begin{tabular}{cc}
\hline Output & RMSE \\
\hline $\mathrm{T}_{1}$ & 0.11 \\
$\mathrm{~T}_{2}$ & 0.20 \\
$\mathrm{~T}_{4}$ & 0.10 \\
$\mathrm{EXT}_{1}$ & 5.47 \\
$\mathrm{EXT}_{2}$ & 5.84 \\
$\mathrm{EXT}_{3}$ & 3.79 \\
$\mathrm{EXT}_{4}$ & 35.63 \\
Overall & 12.79 \\
\hline
\end{tabular}

\section{The Future: SIISEC System}

The results attained by the prediction models for structures behavior open room for the deployment of a system that can monitor in real-time a civil engineering structure (e.g., bridges), based in a Services Oriented Architecture [16], with the following modules, presented in Figure 3:

Data entry: capture all the data using remote sensors, and mobile systems for inspections variables introduced by the specialists, and store them in a transactional database;

Intelligent Monitoring: this module uses the DM module, since the predicting modules should be invoked in a continuous way for online monitoring. In case of abnormal values a set of alerts should be displayed, and the alert(s) should be delivered for the system managers by mobile systems.

Intelligent Decision Support: this module implements the necessary functions to help managers to take decisions using the information available in the system, concerning the bridge security and interventions plans. It implements the tools for reporting and uses the DM module for predicting models invocation and to overview their historic performance.

Data Mining: this module should implement mechanisms for models invocation, and functions to adapt the existing DM models with the new data collected. The results of the invocation and evolution should be stored in the knowledge database, enabling the performance analysis. 


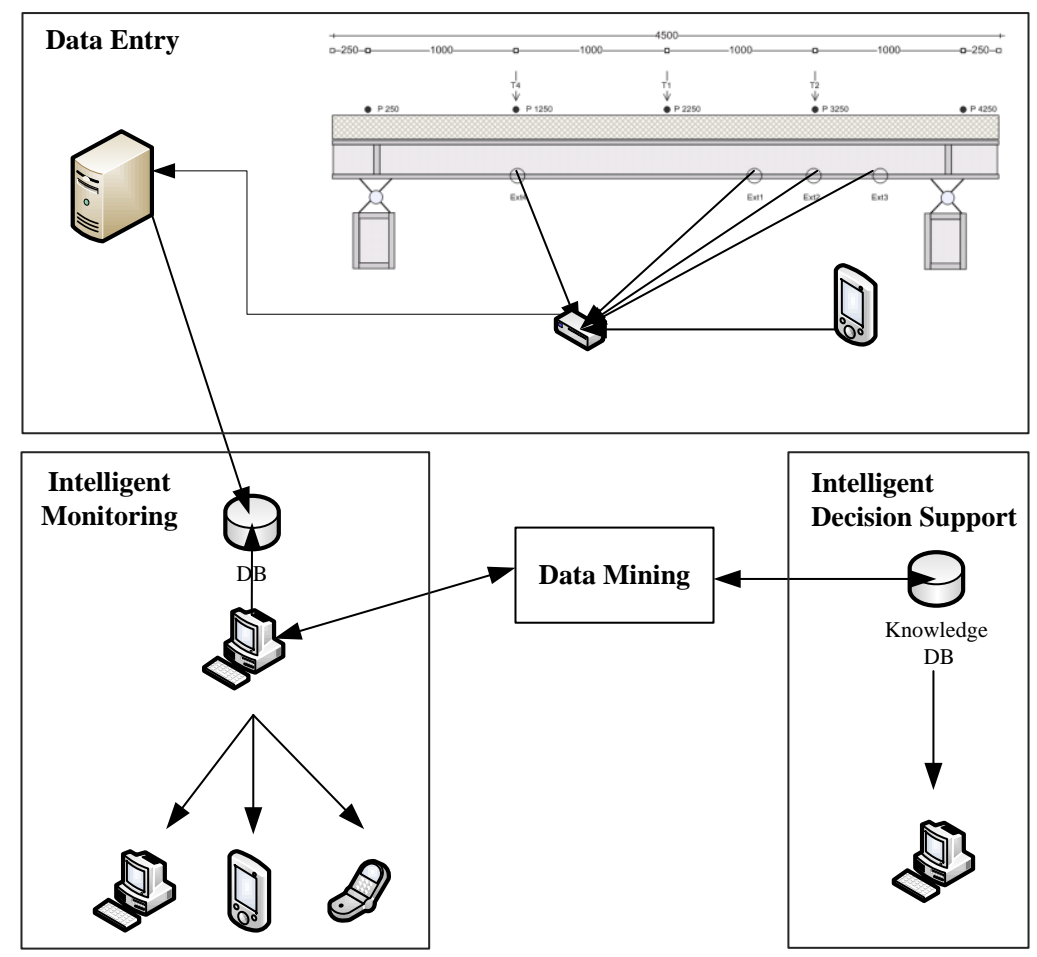

Fig. 3. SIISEC system architecture.

\section{Conclusions}

This paper presents an overview of some experiments that were done in order to develop a real-time Intelligent Decision Support System for civil structures monitoring and behavior prediction. The results attained in the past with simple elements (e.g. beams) opened room for the development of prediction models of deformation and dislocation for a bridge structure prototype with encouraging results, as presented in this study.

Bridge Management is in the starting phase, but there are a lot of works in this area worldwide on inspection and health monitoring, capacity assessment, life-cycle cost analysis and maintenance repair. As proved in the experiments in this study, there are space for the use of techniques from Artificial Intelligence in this kind of systems, to improve the accuracy of deterioration models to predict the future condition sate and safety, in an accurate, fashion and economic way.

In the future we intend to use: data from multiple sources (e.g., traffic data, costs, predicted future conditions) and smart structures that enables a non costly and realtime monitoring of more parameters that can be used to build more reliable systems, 
and improve the accuracy of the proposed SIISEC system, that will be developed in the next phase of this project.

One of the big improvements that should be considered in future versions of the SIISEC system is the adoption of Grid technologies [17] for operation in distributed environments.

\section{References}

1. Casas, J.R., Bridge Management. Actual and Future Trends, Bridge Maintenance, Safety, Management Life-Cycle Performance and Cost - Cruz, Frangopol \& Neves (eds), 2006.

2. Thompson, P.D., Johnson, M.B., Markovian bridge deterioration: deve-loping models from historial data, Structure and Infrastructure Engineering, 1(1): 85-91.

3. Dasgupta, S., Vankayala, V.K., Developing Real Time Business Intelligence Systems: The Agile Way, 2007 1st Annual IEEE Systems Conference, USA, 2007.

4. Michalewicz, Z., Schmidt, M., Michalewicz, M., Chiriac, C., Case Study: An Intelligent Decision Support System, IEEE Intelligent Systems, Vol.20, No . 4, pp. 4449, 2005.

5. Roberts, T. M., Newark, A.C.B., Strength of Webs Subjected to Compressive Edge Loading, Journal of Structural Engineering, American Soc. Of Civil Engineers, 123, N. ${ }^{\circ} 2,176-183,1997$.

6. Santos, M., Quintela, H. Cruz, P., Forecasting of the ultimate resistance of steel beams subjected to concentrated loads using data mining techniques, Proceedings of the Fourth International Conference on Data Mining, Brazil, 2003.

7. Quintela, H., Sistemas de Conhecimento Baseados em Data Mining; Aplicação à Análise da Estabilidade de Estruturas Metálicas, Msc Thesis, University of Minho, 2005.

8. Heaton, J., Understanding the Kohonen Neural Networks, Introduction to Neural Networks with Java, Heaton Research, Inc., 2005.

9. Cruz, P.J.S., Lourenço, L., Santos, M., Quintela, H., Cortez, P., Strength of corroded tapered plate girders under pure shear, Bridge Maintenance, Safety, Management Life-Cycle Performance and Cost - Cruz, Frangopol \& Neves (eds), 2006.

10. Zárate, V.A., Un modelo para el dimensionamento de vigas armadas de inércia variable de alma esbelta, Ph.D. Thesis, Departamento de Ingeniería de la Construcción, Universitat Politécnica de Catalunya, Barcelona, Spain, 2002

11. Quinlan, J.R., Bagging Boosting and C4.5, Proceedings of Fourteenth National Conference on Artificial Intelligence, 1996.

12. Pompe, P., Feelders, A., Using Machine Learning, Neural Networks, and Statistics to Predict Corporate Bankruptcy, Microcomputers in Civil Engineering 12, 267-276, 1997.

13. Cerrito, P.B., Introdution to Data Mining Using SAS Enterprise Miner, SAS Publishing, 2007.

14. Matignon, R., Neural Network Modeling using SAS Enterprise Miner, AuthorHouse, 2005.

15. Souza, J., Matwin, S., Japkowicz, N., Evaluating Data Mining Models: A Pattern Language, Proceedings of the 9th Conference on Pattern Language of Programs, USA, 2002. 
16. Newcomer, E., Lomow, G., Understanding SOA with Web Services, Independent Technology Guides, Addison-Wesley Professional, 2004.

17. Foster, I., Kesselman, C., The anatomy of the grid: Enabling scalable virtual organizations, S. Tuecke, Int. J. Supercomput. Appl. 15 (3), 2001. 